\title{
Research on Space Motion Trajectory Optimization of the Industrial Robot
}

\author{
Gongxing Chen ${ }^{1}$, Luxin Tang ${ }^{2}$ \\ ${ }^{1}$ School of Information and Automation, Guangdong Polytechnic of Science and Trade, Guangzhou, China \\ ${ }^{2}$ Guangdong Engineering Technology Research Center of Industrial Robot Integration and Application, Guangzhou Institute of Science and \\ Technology, Guangzhou, China
}

Email address:

1638839862@qq.com (Gongxing Chen), tangluxin@21cn.com (Luxin Tang)

\section{To cite this article:}

Gongxing Chen, Luxin Tang. Research on Space Motion Trajectory Optimization of the Industrial Robot. Science Journal of Circuits, Systems and Signal Processing. Vol. 10, No. 1, 2021, pp. 10-14. doi: 10.11648/j.cssp.20211001.12

Received: November 13, 2020; Accepted: March 18, 2021; Published: April 16, 2021

\begin{abstract}
In this paper, an online iterative learning planning method is proposed for industrial robot joint space manipulator to capture moving objects. The dynamic mathematical model of the robot is established, and the trajectory of the robot manipulator is tracked and adjusted through the path planning algorithm to change the visual impedance of the robot and optimize the system parameters. It makes the velocity direction of the end effector of the manipulator consistent with the tangent direction of the weld, and realizes the tracking and recognition of the weld trajectory at the end of the manipulator, so as to improve the accuracy and reliability of the robot in capturing moving objects. The simulation results show that the algorithm has good convergence and robustness.
\end{abstract}

Keywords: Industrial Robots, Path Planning, Optimization

\section{Introduction}

Manufacturing industry is the pillar industry of our national economy. "Made in China 2025" clearly points out, China is still in the process of industrialization, and there is a big gap compared with the advanced countries. The manufacturing industry is large but not strong, the independent innovation ability is weak, the key core technology and high-end equipment are highly dependent on foreign countries, and the manufacturing industry innovation system with enterprises as the main body is not perfect. Product grade is not high, lack of world famous brand. The utilization efficiency of resources and energy is low, and the problem of environmental pollution is prominent. Industrial structure is unreasonable, high-end equipment manufacturing and producer services have lagged behind. The level of informatization is not high, and the integration with industrialization is insufficient. The degree of industry internationalization is not high, and the global operation ability of enterprises is not enough.

A control algorithm of human-machine cooperative robot is proposed, which can adjust the admittance damping parameters on line according to the change of environment stiffness [1]. The algorithm first discusses the estimation method of environmental stiffness, which is the basis of operator intention judgment. On this basis, the damping parameters in the mobility controller are changed proportionally to make the robot move more in accordance with the intention of the operator.

A trajectory tracking control method for multi robot system is proposed [2]. Each robot can form a desired formation quickly and track the desired trajectory according to the desired formation.

With regard to the uncertainty of the target motion and the unknown of the system to the target motion mode, a multi-mode filtering target tracking algorithm based on suboptimal parallax is proposed [3]. Firstly, the direction of the largest change of the ellipsoid projection area is the suboptimal parallax direction, which is used as the parallax control direction of the robot. Secondly, the probability of various motion modes of the target is calculated by the multi-mode filtering algorithm [4-7]. Then, the target states of each motion mode are estimated. Finally, the target states are estimated by the probability weighting of each motion mode.

Therefore, in this paper, the online iterative learning 
algorithm is used to update the rules of weights, so that the online learning objective function is minimized, and the robot end actuator trajectory is optimized. There are three tasks to realize the automatic tracking of the curve welding seam for the 6-DOF welding robot as below. One is to ensure that the welding gun can track the welding seam with high precision. The other is to ensure that the welding gun can carry out the welding in the desired attitude. The last is to ensure that the welding robot can track the welding seam with high precision In the process of visual tracking of curved weld seam, the image coordinates of the weld point are within the acceptable range of the expected image coordinates, ensuring that the angle between the weld direction and the laser strip is close to 90 degrees, ensuring that the image with high quality can be obtained and the tracking quality can be improved.

\section{Robot Structure Analysis}

Industrial robot is composed of robot body, control cabinet and teaching box, which are connected by cables. The specific structural parameters are shown in Table 1 . The robot measures the contour of the object by laser, processes the contour image, and transforms the laser image coordinate to the robot coordinate. The robot controller adjusts and realizes the trajectory of the robot according to the offset value.

As shown in Figure 1, the transformation between the start point and the end point is decomposed into one translation and two rotations. Translation is to move the coordinate origin from the start point to the end point, the first rotation is to align the end actuator coordinate system with the desired attitude, and the second rotation is to turn the end actuator around its own axis to the final attitude [8-10]. Moreover, the generated value of the space trajectory planning function in rectangular coordinates is the position and orientation of the robot end effector shown in Figure 2.

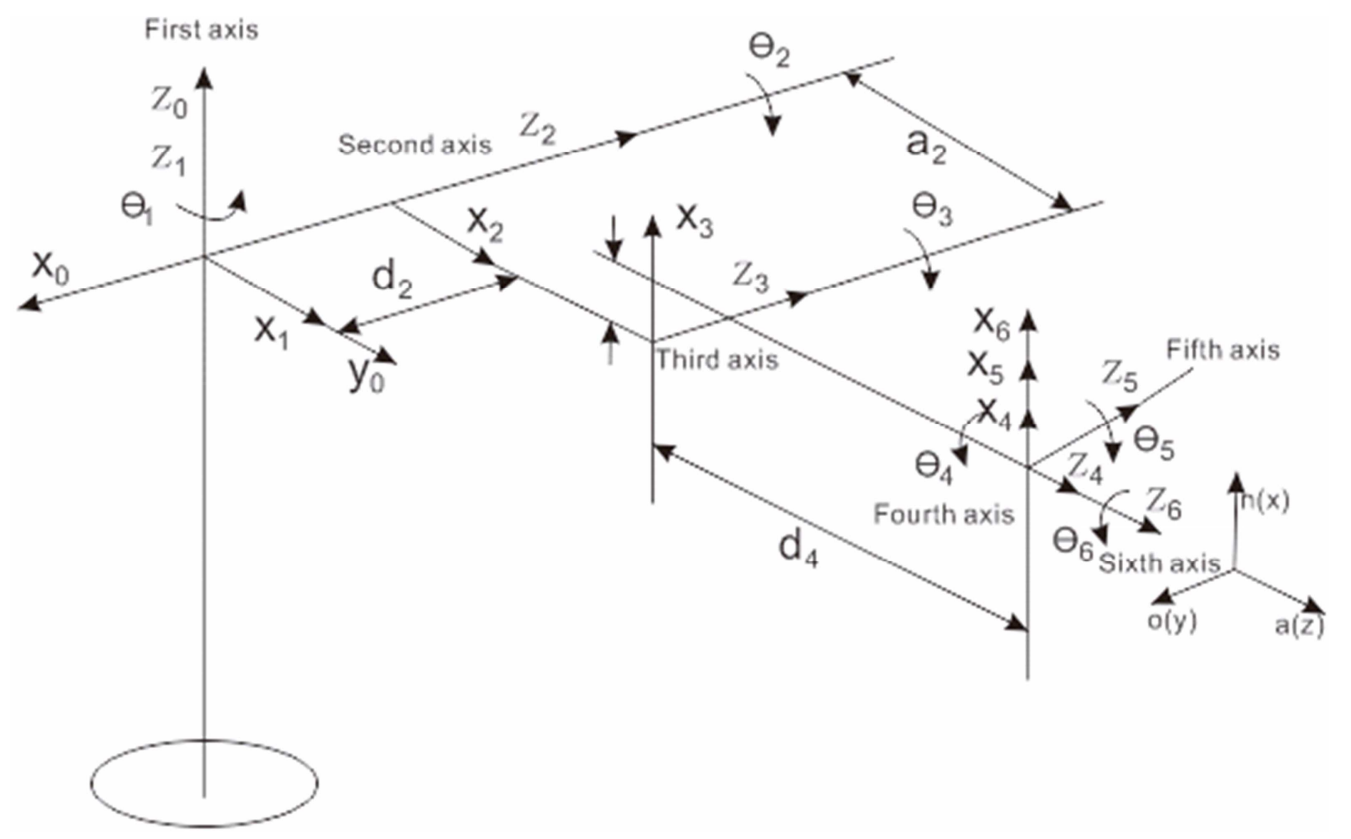

Figure 1. Structure of industrial robot.

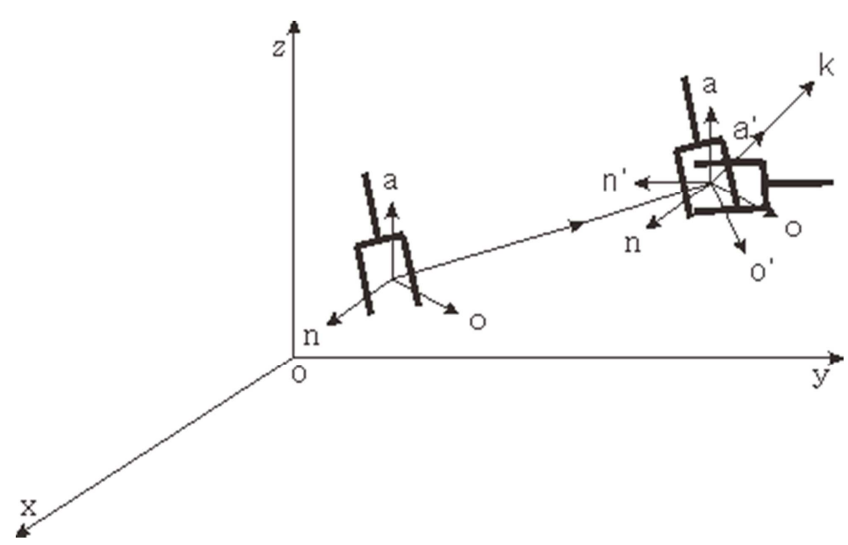

Figure 2. Exchange between start point and end point in Cartesian space trajectory planning
Table 1. Structural parameters of industrial robot.

\begin{tabular}{lll}
\hline Sequence numbers & Names & Parameters \\
\hline One & Structural type & Articulated robot \\
Two & Degrees of freedom & six \\
Three & Payload & $4 \mathrm{KG}$ \\
Four & Maximum active radius & $630 \mathrm{~mm}$ \\
Five & Repeated positioning accuracy & $\pm 0.05 \mathrm{~mm}$ \\
Six & J1 axis Motion angle & $\pm 165^{\circ}$ \\
Seven & J2 axis Motion angle & $-80^{\circ},+130^{\circ}$ \\
Eight & J3 axis Motion angle & $-130^{\circ},-208^{\circ}$ \\
Nine & J4 axis Motion angle & $\pm 150^{\circ}$ \\
Ten & J5 axis Motion angle & $\pm 135^{\circ}$ \\
Eleven & J6 axis Motion angle & $\pm 360^{\circ}$ \\
Twelve & Actual weight of body & $45 \mathrm{KG}$ \\
Thirteen & Temperature & $0-45^{\circ} \mathrm{C}$ \\
Fourteen & Humidity & $20-80 \% \mathrm{RH}$ \\
Fifteen & Vibration & $4.9 \mathrm{~m} / \mathrm{s}^{2}$ below \\
Sixteen & Power capacity & $2.95 \mathrm{KVA}$ \\
\hline
\end{tabular}




\section{Robot Dynamics Modeling}

In order to control the industrial robot accurately, the kinematic model and dynamic model of the robot must be established. Robot kinematics mainly studies the relationship between the spatial displacement of robot joint variables and the position and orientation of robot end effector [11-13]. Robot dynamics mainly studies the forces and moments generated when the robot joints interact with the external environment.

The dynamic model equation of the robot is expressed as

$$
\mathrm{M}(q) \ddot{q}+h(q, \dot{q})=\tau-J^{T} F
$$

Where, $q$ is the robot joint variable, $\tau$ is the control moment, $F$ is the operating force vector of robot end actuator, $h(q, q)$ is the combined vector of centrifugal force and gravity of robot, $\mathrm{M}(q)$ is the positive definite matrix of inertia parameters in $n$-dimensional symmetry. It satisfies the relationship as follows.

$$
\lambda_{\min } \leq M(q) \leq \lambda_{\max }
$$

Among them, $\lambda_{\min }, \lambda_{\max }$ as the minimum and maximum eigenvalue of $\mathrm{M}(q)$ in all joint configurations.

The control torque of the robot end actuator can be expressed as

$$
\tau=\mathrm{M}(q) J^{-1}(q)(\ddot{r}-\dot{J} \dot{q})+h(q, \dot{q})+J^{T} F
$$

The manipulator arm of the robot is connected by a series of connecting rods through rotating or moving joints. Each joint is driven by a servo motor, and the relative motion of the joints results in the motion of the connecting rods.

The joint torsion $\alpha_{i}$, link length $t_{i}$, adjacent links between joint variable translation $d_{i}$ and joint angle $q_{i}$ are described by the joint variable parameters based on GSK six axis industrial robot in Table 2.

Table 2. Structural parameters of robot joint.

\begin{tabular}{lllll}
\hline joint & $\alpha_{i} /$ (degree) & $\boldsymbol{\eta} / \mathbf{m m}$ & $d_{i} / \mathbf{m m}$ & $q_{i}$ (degree) \\
\hline 1 & -90 & 0 & 0 & 0 \\
2 & 90 & 0 & 215 & 0 \\
3 & -90 & 0 & 0 & 0 \\
4 & 90 & 0 & 308 & 0 \\
5 & -90 & 0 & 0 & 0 \\
6 & 0 & 0 & 265 & 0 \\
\hline
\end{tabular}

The pose of the end effector of six axis industrial robot in Cartesian coordinate system is expressed as

$$
r=(x, y, z, \theta, \varphi, \phi)^{T}
$$

The corresponding joint variable is

$$
q=\left(q_{1}, q_{2}, q_{3}, q_{4}, q_{5}, q_{6}\right)^{T}
$$

The functional relationship between the pose of the robot end effector and the joint variables is expressed as

$$
r=f(q)=\left(f_{1}(q), f_{2}(q), \ldots, f_{6}(q)\right)
$$

The expression of the relationship between the velocity of the robot end effector and the velocity of the joint is

$$
\begin{gathered}
\dot{r}=J(q) \dot{q} \\
J=\frac{\partial f(q)}{\partial q^{T}}=\left[\begin{array}{ccc}
\frac{\partial f_{1}}{\partial q_{1}} & \cdots & \frac{\partial f_{1}}{\partial f_{6}} \\
\vdots & & \vdots \\
\frac{\partial f_{6}}{\partial q_{1}} & \cdots & \frac{\partial f_{6}}{\partial q_{6}}
\end{array}\right]
\end{gathered}
$$

The Jacobian matrix $\mathrm{J}$ of the robot changes with the position and the pose of the end effector, changes the speed of the robot end actuator and the speed of the joint [14-15].

The velocity of each joint is coordinated to ensure that the end actuator moves stably in the desired Cartesian coordinate direction. The acceleration of the end actuator is

$$
\ddot{r}(t)=\dot{J}(q, \dot{q}) \dot{q}(t)+J(q) \ddot{q}(t)
$$

The controlled equation of joint velocity and acceleration is

$$
\begin{array}{r}
\ddot{q}(t)=J^{-1}(q) \ddot{r}(t)-J^{-1}(q) \dot{J}(q, \dot{q}) J^{-1}(q) \dot{r}(t) \\
\ddot{r}(t)=\ddot{r}_{d}(t)+k_{1}\left(\dot{r}_{d}(t)-\dot{r}(t)\right)+k_{2} e(t)
\end{array}
$$

Where, $\mathrm{e}(\mathrm{t})$ is the pose error function of the end effector, $\mathrm{K} 1$ and $\mathrm{K} 2$ are the velocity error coefficients, pose error coefficients of the end effector separately, and $\ddot{r}_{d}(t)$ is the desired acceleration of the robot.

When the parameters of each link are determined, the joint transformation matrix among adjacent links can be obtained as

$$
P_{i}^{i-1}=\left[\begin{array}{cccc}
\cos q_{i} & -\sin q_{i} & 0 & \boldsymbol{l}_{i-1} \\
\sin q_{i} \cos \alpha_{i-1} & \cos q_{i} \cos \alpha_{i-1} & -\sin \alpha_{i-1} & -\sin \alpha_{i-1} d_{i} \\
\sin q_{i} \sin \alpha_{i-1} & \cos q_{i} \sin \alpha_{i-1} & \cos \alpha_{i-1} & \cos \alpha_{i-1} d_{i} \\
0 & 0 & 0 & 1
\end{array}\right]
$$

Therefore, through the transformation matrix of each joint, the relationship between the base coordinate system and the end actuator coordinate system of the robot can be obtained as follows.

$$
P_{m}^{0}=P_{1}^{0} P_{2}^{1} \cdots P_{m}^{m-1}
$$

\section{Path Planning Algorithm and Optimization}

Trajectory tracking control is to make the robot end move along the planned path with a definite pose. The error and error rate of each joint of industrial robot is an input space. Online iterative learning algorithm can be expressed as 


$$
W_{n m}(k+1)=W_{n m}(k)+\frac{a_{n m}}{N_{e}} A_{n m}(k)\left[f_{m}^{v}(k)-A_{n m}^{T}(k) W_{n m}(k)\right]
$$

Where, $w_{n m}(k+1)$ is the associative strength of the n-th input space for the m-th output at $(\mathrm{k}+1)$ time; $A_{n m}(k)$ is the activation vector of the $\mathrm{n}$-th input space for $w_{n m}(k)$ at $\mathrm{k}$ time; $f_{m}^{v}(k)$ is the expected value of the $\mathrm{m}$-th output at $\mathrm{k}$ time; $a_{n m}$ is the online learning rate; $N_{e}$ is the number of storage units.

By using online iterative learning algorithm, the rules of weights are updated continuously to minimize the online learning objective function $\mathrm{J}$, and the robot end actuator is close to the moving object.

$$
\begin{aligned}
\mathrm{F}_{m}^{v}(k+1) & =F_{m}^{v}(k)-\eta \frac{\partial J_{o n}}{\partial F_{m}^{v}} \\
J_{o n} & =\frac{1}{2} F^{T} F \\
F_{s}^{V}=A_{s}^{T} W & =\left(f_{s 0}^{v}, f_{s 1}^{v}, \ldots f_{s 5}^{v}\right)
\end{aligned}
$$

Where, the input space $S=[\Delta x, \Delta x, \Delta x]$ is the position difference between the moving targets of the end effector in Cartesian coordinate system; $J_{o n}$ is the online learning objective function; $\mathrm{W}$ is the weight vector of the input space;
$\mathrm{F}_{m}^{v}(k+1)$ is the expected value of the moving targets of the robot end effector at the $\mathrm{K}+1$ moment; $\eta$ is the learning rate.

In order to ensure the stability of the system and improve the control performance, the compensation control input is added as

$$
\tau=\alpha \mathrm{F}^{p}+(1-\alpha) \mathrm{F}^{\mathrm{v}}=\mathrm{J}^{\mathrm{T}}\left[\alpha \mathrm{MU}+\alpha h+(1-\alpha) \mathrm{F}^{v}\right]
$$

Where, $\alpha$ is the compensation control factor, $\mathrm{F}^{p}$ is the output of the position controller, $\mathrm{F}^{\mathrm{v}}$ is the output of the visual impedance feedback.

\section{Simulation Verification of Robot trajectory}

In the motion trajectory, $\mathrm{S}=[\Delta x, \Delta x, \ddot{\Delta x}, \Delta y, \Delta y, \ddot{\Delta y}, \Delta z, \Delta z, \ddot{\Delta z}]$ that is the position state vector is input to transform the position and attitude trajectory of the robot end effector into the trajectory in joint space. After the forward kinematics calculation of the smooth trajectory, the original target trajectory in Cartesian coordinates is optimized to the desired position of the end actuator corresponding to the joint variable in Cartesian coordinates.
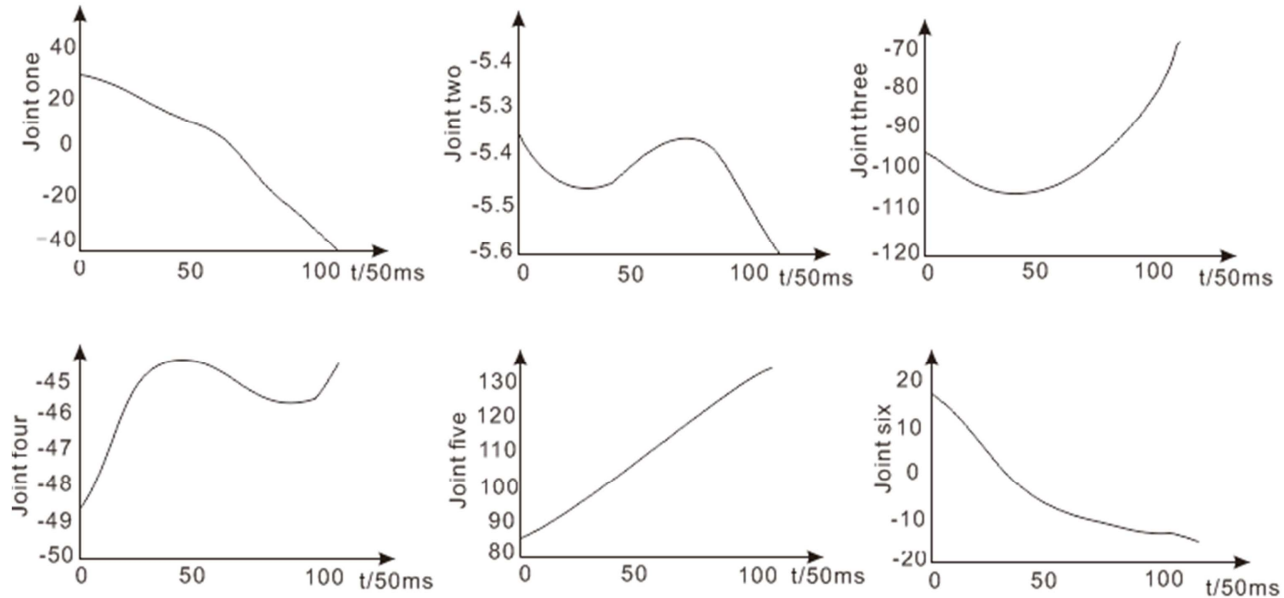

Figure 3. Track curve of moving target in joint coordinate system

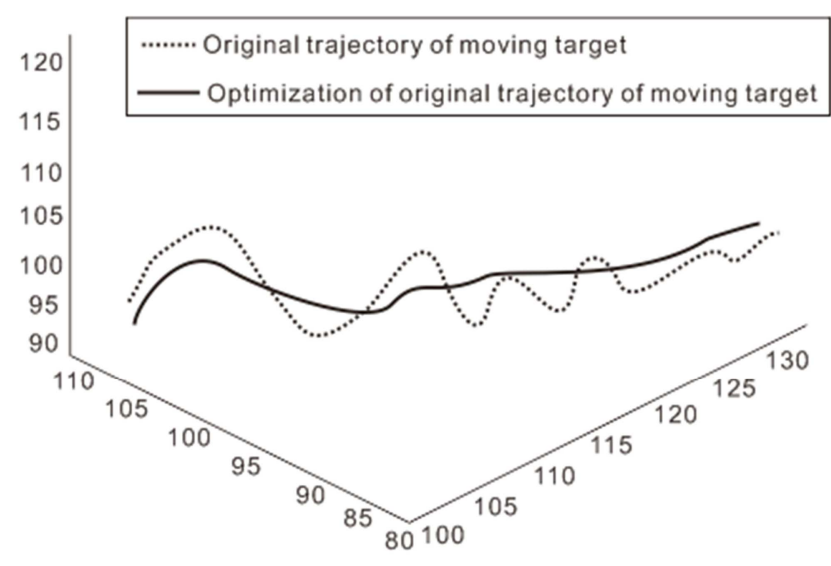

Figure 4. Track curve of moving target in joint coordinate system.
In the process of moving track, when $\eta$ is 0.98 and $\tau$ is 0.63 , the size of visual impedance is adjusted and tracked by on-line iterative algorithm. In the case of no input compensation, the result of calculation of visual impedance control quantity is directly carried out. As the end effector of the robot approaches the moving target, the visual impedance gradually decreases until the end effector of the robot finishes the task of target grasping. The simulation results after network compensation. It can be seen that better impedance trace can be obtained by compensation in Figure 4.

\section{Acknowledgements}

This work is partially supported by Industrial robot intelligent production line and guangdong industrial robot 
integration and Application Technology Engineering Research Center. The authors also gratefully acknowledge the helpful comments and suggestions of the reviewers, which have improved the presentation.

\section{References}

[1] Wang Fei, Chao Zhiqiang, Zhang Chuanqing, Li Huaying. Simulation of variable mobility control of robot arm trajectory tracking [J]. Computer simulation 2018, 35 (12): 280-285.

[2] Chen Mei, Ding Minggang, Zheng Tao. Trajectory tracking control of multi robot system based on navigation following method [J]. Control engineering, 2019, 26 (01): 37-42.

[3] Huang Shuai, Fu Guangyuan, Wu Ming, Yue min. Multi mode filtering target tracking algorithm based on monocular suboptimal disparity in unknown environment [J]. Computer application 2019, 39 (03): 864-868.

[4] Zeng Xiangxin, Cui Naigang, Guo Jifeng. Path planning of space robot based on HP adaptive pseudospectral method [J]. Robot 2018, 40 (30): 385-392.

[5] Cao Qihe, Li Qinghua, Qiu Shubo, Han Fengjian, Feng Chao. Partition sampling strategy for robot motion planning under uncertainty $[\mathrm{J}]$. The Journal of China Universities of Posts and Telecommunications, 2020, 12.

[6] Li Xueyong, Zhao Zhongqiu, Zhang Chunsong, Lu Changhou. Calculation method of human manipulator interaction force based on finite element method [J]. Journal of Jilin University (Engineering Edition), 2020, 23: 27-30.

[7] Yang Hongguo, Gu Lifen. Research on global path planning of agricultural robot based on Hybrid Particle Swarm Optimization [J]. Research on Agricultural Mechanization, 2021, 43 (10): 33-36.
[8] Zhang Yuanxun, Liu Yingbo, Gu Chengpeng, Du Xuesong, Huang Fan. Gait planning and analysis of six wheeled legged robot in unstructured environment under typical road conditions [J]. Mechanical science and technology, 2020, 12.

[9] Xu Mengying, Wang Jiaojiao, Liu Baoma, Liang Cailin, Jie Xiangli, Zhoujie. Gait planning and analysis of six wheeled legged robot in unstructured environment under typical road conditions [J]. Robot path planning based on Improved Genetic Algorithm. Journal of Shihezi University (NATURAL SCIENCE EDITION), 2020, 12.

[10] Yang Jing, Wang Qingyun, Liu Erwei, Zhang Zhuo. Trajectory planning and simulation analysis of $3 \mathrm{R}$ second lining cutting robot [J]. Southern agricultural machinery, 2020, 23: 17-22.

[11] Yao Gang. Research on trajectory planning of industrial robot handling operation $[\mathrm{J}]$. Southern agricultural machinery, 2020, 23: $27-30$

[12] Zhang Qingsong, Duan Shuaichen, Xia Re. Workspace analysis and Simulation of humanoid manipulator based on MATLAB [J]. Mechanical drive, 2020, 12: 99-105.

[13] Yuan Quan, Lv Xunjie. Research on path planning optimization of mobile car based on PRM algorithm [J]. Journal of Ningbo Institute of Technology, 2020, 04: 31-35.

[14] Marco Manca, Fabio Paternò, Carmen Santoro, Eleonora Zedda, Chiara Braschi, Roberta Franco, Alessandro Sale. The impact of serious games with humanoid robots on mild cognitive impairment older adults [J]. International Journal of Human-Computer Studies, 2020, 12.

[15] Chen Gongxing. Research on robot electrical fault detection and diagnosis based on deep learning [J]. Automation and instrumentation, 2020, 35 (12): 40-44. 